\title{
Examen de la regularidad de crudos y precisión del espectrómetro multicanal de rayos $X$
}

J. ESS. ERLANGEN

Zement Kalk Gips, $n^{\circ} 1$, enero 1970, págs. 23-31

\section{INTRODUCCION}

Para una buena calidad del clínker es decisivo un crudo con propiedades químicas y físicas constantes. Las propiedades físicas - por ejemplo la distribución granulométrica - se pueden mantener constantes con relativa facilidad y no serán objeto en estas aclaraciones. Las propiedades químicas - proporciones de los óxidos $\mathrm{CaO}, \mathrm{SiO}_{2}, \mathrm{Al}_{2} \mathrm{O}_{3}$ y $\mathrm{Fe}_{2} \mathrm{O}_{3}$ en la mezcla total-son mucho más difíciles de mantener dentro de límites de tolerancia calculados previamente. Por consiguiente, es indispensable un control de la composición química en las diversas etapas de la preparación. Los materiales que suministra la cantera fluctúan irregularmente en su composición química. En consecuencia, valores aislados de la composición química son menos interesantes que las propiedades de un gran número de valores aislados. Las reglas para dictaminar tales grupos de valores de medición los suministra la estadística matemática.

Con las leyes de lá estadística matemática se iniciaron el pasado año extensos exámenes de la instalación de molienda de crudo en una fábrica de cemento alemana (1). Durante el mes de mayo del presente año se realizó una nueva serie de ensayos. Debían de confirmar los resultados de los ensayos anteriores y suministrar más aclaraciones.

Ahora se dispone del material numérico. En los apartados siguientes se explica la calidad de la regulación de la mezcla, las propiedades del material crudo después de los silos de almacenamiento y la precisión que en la explotación se espera del espectrómetro multicanal de rayos $\mathrm{X}$, tomando como base estas cifras.

\section{PUNTOS DE TOMA DE MUESTRAS}

Los diversos puntos de toma de muestras y la técnica empleada los ha descrito Gerstberger detalladamente (1). Por lo tanto, ahora sólo se describirán brevemente las estaciones esenciales de la preparación del crudo (fig. 1).

El material crudo llega, a través de la trituración previa, directamente de la cantera a los silos de grava. Los componentes cal y arcilia sólo acusan fluctuaciones reducidas en su composición química y por su cantidad son un material adicional. La proporción mayor en la mezcla cruda la constituye la marga, la cual fluctúa notablemente en su composición. 
Desde los silos de grava los diferentes componentes llegan, a través de básculas dosificadoras de cinta, al molino de crudo. La primera toma de muestras se efectúa después del molino en el punto de toma 1 , y la segunda en el punto de toma 3 , inmediatamente delante del horno.

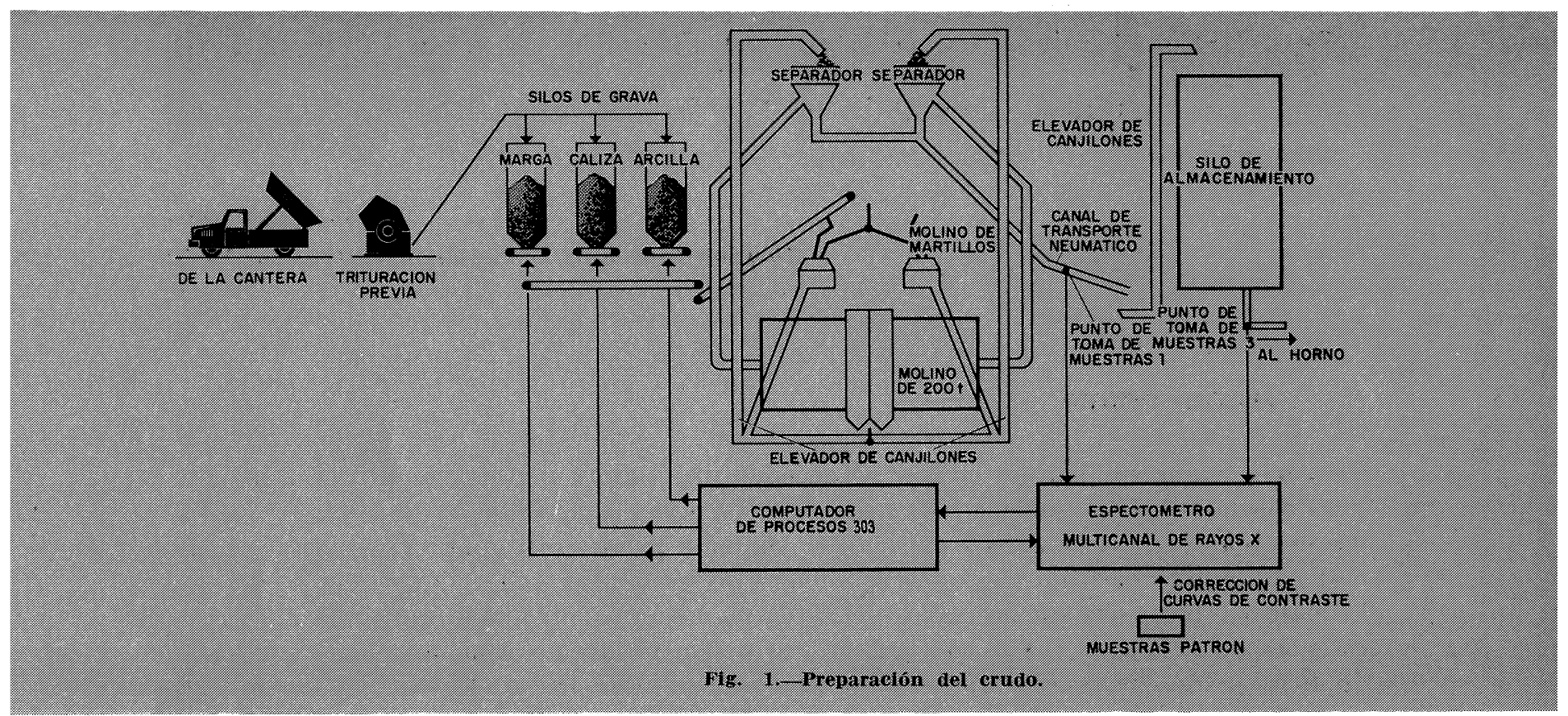

$\mathrm{El}$ análisis se realiza con un espectómetro multicanal de rayos $\mathrm{X}$.

Los análisis se producen en forma de contador de impulsos que el computador transforma en las correspondientes concentraciones.

\section{PRECISION DE LA REGULACION DE LA MEZCLA}

\subsection{Filosofía de la regulación de la mezcla}

La regulación de la mezcla ha sido descrita detalladamente por Gerstberger (2) y. Wackerle (3). Por lo tanto, aquí sólo se resumirán brevemente las propiedades fundamentales.

En la regulación de la mezcla se trata de una regulación DDC (regulación digital directa). La muestra se toma en el punto 1 en un retículo de tiempo fijo (30 minutos) y se la analiza con el espectrómetro de rayos $\mathrm{X}$. Por el contador de impulsos el computador de procesos calcula las concentraciones de $\mathrm{CaO}, \mathrm{SiO}_{2}, \mathrm{Al}_{2} \mathrm{O}_{3}$ y $\mathrm{Fe}_{2} \mathrm{O}_{3}$ de la mezcla actual. Esta mezcla está constituida por dos ingredientes conocidos, o sea cal y arcilla, y un ingrediente desconocido, la marga. Entre la composición de la mezcla en las básculas dosificadoras de cinta y la toma de muestras, existe un ciclo de toma de muestras, el tiempo durante el cual el material circula por el molino. Si se supone que el ingrediente desconocido varía poco durante dos ciclos de toma de muestras, de una parte, y a base de los conocidos ajustes de las básculas y de los áridos conocidos cal y arcilla, es posible calcular la composición química del ingrediente desconocido marga, y de la otra, puede indicarse un nuevo ajuste de las básculas, con lo que se puede hacer coincidir el valor standard de la cal que se persigue $\mathrm{KSZ}$ con el valor efectivo $\mathrm{KS}$. 
El balance de los anteriores valores efectivos sumados con el valor nominal $\mathrm{KS}_{\text {nom }}$ proporciona el valor que se persigue KSZ para un número subsiguiente $\mathrm{n}$ de ciclos de toma de muestras, según la relación:

$$
\mathrm{KSZ}_{\mathrm{i}}=\mathrm{KS}_{\mathrm{nom}}+\mathrm{KSZ}_{\mathrm{i}-1}-\frac{\sum_{\mathrm{k}=1}^{\mathrm{n}} \mathrm{KS}_{\mathrm{k}}}{\mathrm{n}}
$$

en la cual:

$\mathrm{n}$ es el número de ciclos;

$\mathrm{i}, \mathrm{k}$, los índices contadores.

\subsection{Comparación de la concentración del óxido de calcio y del anhídrido silícico con y sin regulación}

\subsection{Algunos conceptos de la estadística matemática}

Para entender más fácilmente las siguientes explicaciones, se expondrán algunos conceptos de la estadística matemática (4) que se utilizarán más adelante.

El valor medio $\overline{\mathrm{x}}$ se calcula de $\mathrm{N}$ valores aislados según la fórmula:

$$
\bar{x}=\frac{1}{N}\left(x_{1}+x_{2}+\ldots \ldots . .+x_{N}\right)=\frac{1}{N} \sum_{i=1}^{n} x_{i}
$$

El cuadrado de la dispersión

$$
\mathrm{s}^{2}=\frac{1}{\mathrm{~N}-1}\left[\left(\mathrm{x}_{\mathrm{i}}-\overline{\mathrm{x}}\right)^{2}+\ldots \ldots . .+\left(\mathrm{x}_{\mathrm{N}}-\overline{\mathrm{x}}\right)^{2}\right]=\sum_{\mathrm{i}=1}^{\mathrm{N}} \frac{\left(\mathrm{x}_{\mathrm{i}}-\overline{\mathrm{x}}\right)^{2}}{\mathrm{~N}-1}
$$

tiende, para un número infinito de mediciones, a la varianza $\sigma^{2}$

$$
\lim _{\mathrm{N} \rightarrow \infty}^{\mathrm{S}^{2}}=\sigma^{2}
$$

y representa un valor aproximado de la varianza conforme a lo que se esperaba.

Frecuentemente se emplea el coeficiente de variación

$$
\mathrm{v}=\mathrm{s} / \overline{\mathrm{x}}
$$

La zona de dispersión de una variable estadística $\mathrm{x}$ depende de la certidumbre estadística $\mathrm{S}$ y de la dispersión $\mathrm{s}$. Se caracteriza brevemente por el dato:

$$
\mathrm{x}=\overline{\mathrm{x}} \pm \mathrm{u}(\mathrm{S}) \cdot \mathrm{s}
$$

e indica en qué zona $\mathrm{S} \%$ caen todos los análisis. La magnitud u (S) es el fractil que corresponde a una certidumbre estadística $\mathrm{S}$. 
Los fractiles $\mathrm{u}(\mathrm{S})$ se encuentran como función de la seguridad estadística $\mathrm{S}$ en todas las obras competentes de la estadística matemá tica en forma de tablas [por ejemplo (5)].

En el conjunto básico distribuido normalmente es la media de las pruebas al azar, la que representa una aproximación para el medio de población $\mu$, asimismo distribuido normalmente con una dispersión:

$$
\mathrm{s}_{\text {medio }}=\mathrm{s} / \sqrt{\mathrm{N}}
$$

Con una certidumbre estadística $\mathrm{S}$ dada, el medio de población es cubierto por el intervalo de confianza:

$$
\overline{\mathrm{x}}-\mathrm{u}(\mathrm{S}) \cdot \mathrm{s}_{\text {medio }} \leqslant \mu \leqslant \overline{\mathrm{x}}+\mathrm{u}(\mathrm{S}) \cdot \mathrm{s}_{\text {medio }}
$$

O expresado en otros términos: el medio de populación $\mu$ se halla entre los límites de confianza:

$$
\overline{\mathrm{x}} \pm \mathrm{u}(\mathrm{S}) \cdot \mathrm{s}_{\text {medio }}
$$

\subsection{Los resultados de seis muestras escogidas al azar}

La tabla 1 presenta los resultados de seis muestras tomadas al azar, cada una de 100 análisis aislados, en el punto de toma 1 (fig. 1). Se ha indicado en cada caso para las 4 concentraciones (c, s, $a, \mathrm{f})$ y los 3 módulos (KS, SM, TM) el valor medio $\overline{\mathrm{x}}$, la dispersión s y el coeficiente de variación v.

Con el fin de obtener datos sobre la calidad de la regulación, hay que comparar la explotación regulada con la no regulada. Esta comparación puede hacerse fácilmente en las concentraciones. Las fluctuaciones en la explotación regulada se reproducen directamente por la dispersión d:1 análisis del crudo.

Datos sobre la explotación no regulada se obtienen con ajuste fijo de las básculas y volviendo a analizar la marga. O sea, que si no varía la regulación fija de las básculas dosificadoras de cinta, las fluctuaciones en la composición de la marga pueden repercutir absolutamente en el material crudo. Las dispersiones del análisis de la marga de la tabla 1 se presentarán multiplicadas por el factor de la báscula de la marga.

Los ajustes típicos de las básculas se hallan en $80 \%$ para la báscula de la marga y en $10 \%$ para las básculas de la caliza y de la arcilla. Por lo tanto, el $80 \%$ de los valores de dispersión de la marga en la tabla 1 corresponden a la explotación no regulada.

En las figuras 2 a 4 se representa la gama de dispersión de las concentraciones y los coeficientes de amortiguamiento. Estos indican en que valor se reduce la gama de dispersión en la explotación regulada con respecto a la de la explotación no regulada. Una consideración similar para el standard de cal no es posible en esta forma sencilla, ya que los errores de la concentración no son independientes unos de otros y por esta causa no puede aplicarse la ley de propagación del error. De acuerdo con la experiencia, en el standard de la cal hay que contar con un coeficiente de amortiguación entre 2 y 3.

Como quiera que las muestras tomadas al azar se han tomado directamente unas despues de otras, en las figuras 2 a 4 se han unido los puntos entre sí. 


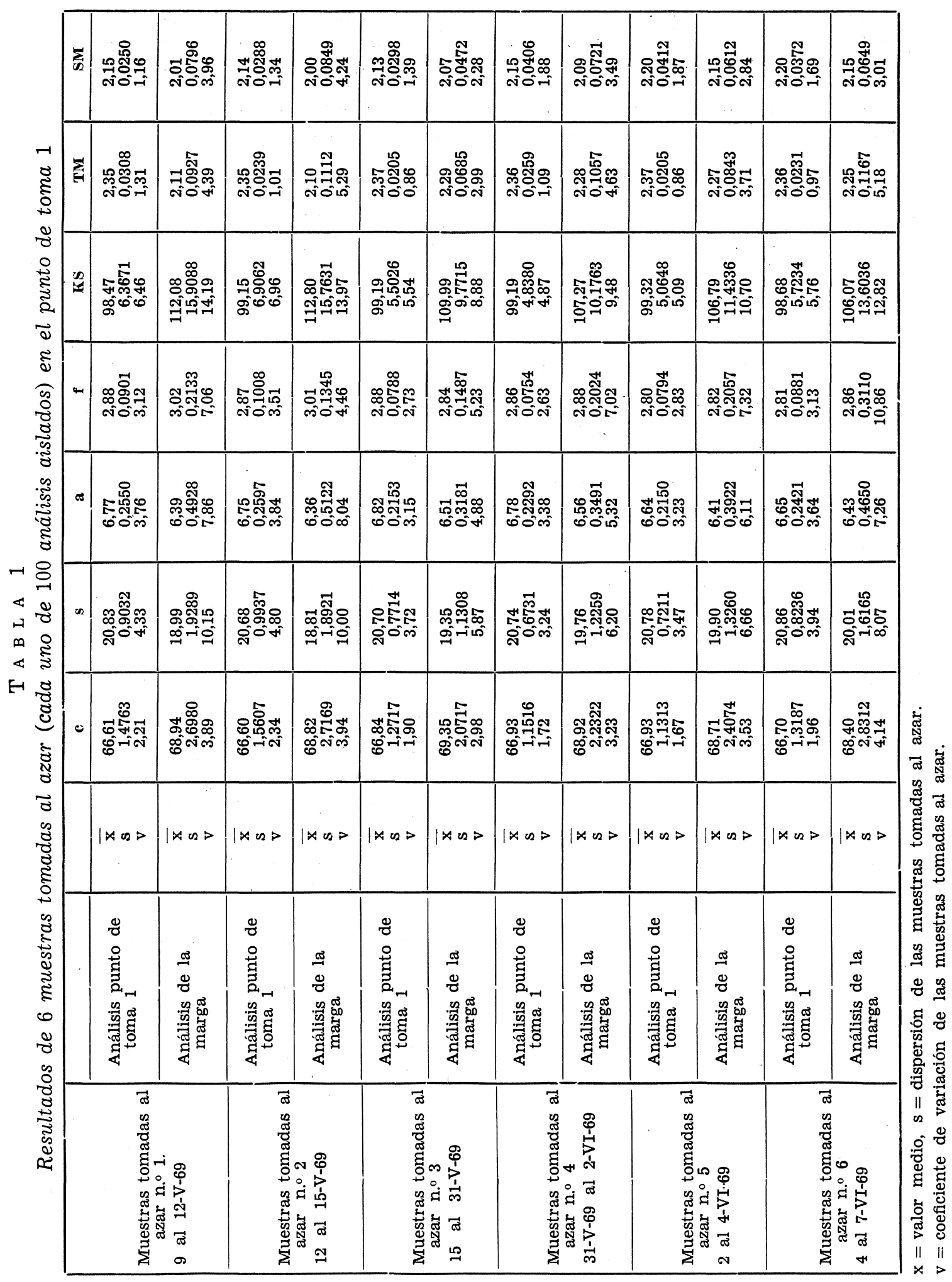

17 
Las figuras 2 a 4 pueden discutirse como sigue:

1. La gama de dispersión y los coeficientes de amortiguamiento dependen de la muestra tomada al azar y del tiempo, ya que en éste se suceden inmediatamente; es decir, que el coeficiente de amortiguamiento de la regulación no es constante.

2. Para pequeñas gamas de dispersión en el tamaño de entrada los factores de amortiguación son menores que para gamas de dispersión grandes.

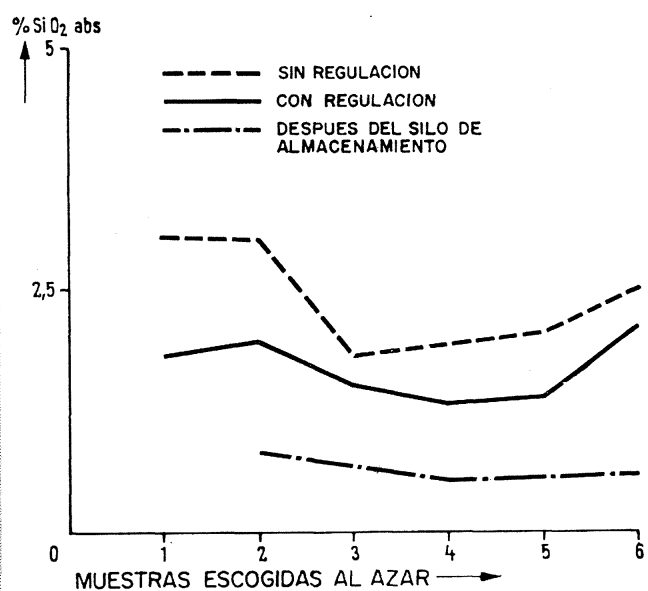

Fig. 2.-Gama de dispersión de las concentraciones de $\mathrm{SiO}_{2}$ para un $95 \%$ de certidumbre estadística.

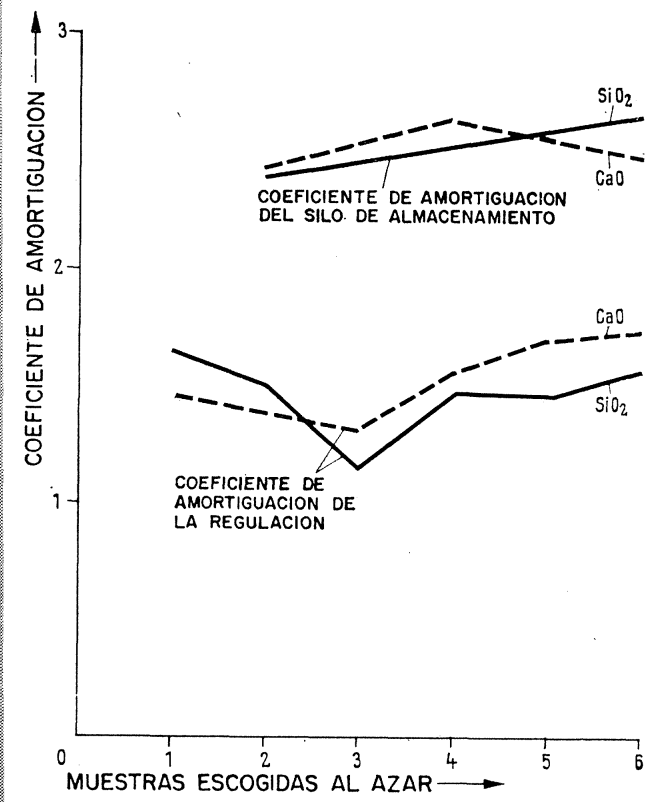

Fig. 4.-Coeficientes de amortiguación para $\mathrm{CaO}$ y $\mathrm{SiO}_{2}$.

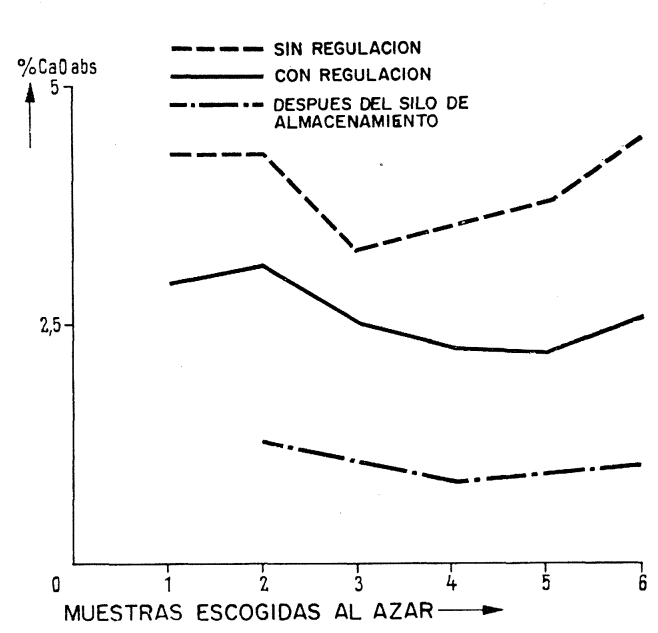

Fig. 3.-Gama de dispersión de las concentraciones de CaO para un $95 \%$ de certidumbre estadística.

Estos dos hechos llevan a la conclusión de que el dictamen de la regulación sólo a base de la dispersión puede llevar a conclusiones erróneas. La dispersión no es ninguna medida suficiente para la calidad de la regulación de la mezcla. Es absolutamente necesario saber con qué velocidad varían las concentraciones de los materiales de partida. Variaciones de baja frecuencia se dominan por la regulación mucho mejor que aquellas que se acercan a las proximidades del ciclo de toma de muestras.

Por este motivo, en primer lugar, se analizarán con más detalle los valores medios obtenidos de cada una de las muestras tomadas al azar.

A continuación se obtendrá, por un análisis de Fourier de valores aislados sucesivos en el tiempo, una afirmación sobre el espectro de frecuencia de explotación regulada y no regulada. 


\subsection{Descomposición de la dispersión y límites de confianza}

La calidad de los valores medios obtenidos puede comprobarse en forma sencilla con la descomposición de la dispersión, que aclara si es lógico el reunir varias muestras tomadas al azar en una muestra al azar común de volumen $\mathrm{N}$ o el considerar cada prueba al azar por separado:

$$
N=\sum_{j=1}^{M} N_{j}
$$

donde:

$\mathrm{N}_{\mathrm{j}}$ es el volumen de la muestra tomada al azar $\mathrm{j}$;

M, el número de muestras tomadas al azar.

Si con esto se consigue demostrar que las muestras tomadas al azar de la tabla 1 en la explotación no regulada, divergen tan fuertemente en el valor medio que hay que tratarlas por separado y que, en cambio en la explotación regulada, pueden reunirse en una muestra al azar común, la regulación habría aportado una mejora notable en los valores medios.

Para hacer esta demostración, hay que completar antes los valores medios y las dispersiones del análisis de la marga con ayuda del ajuste de las básculas.

$$
\begin{gathered}
\text { marga } \\
80 \%
\end{gathered} \frac{\text { cal }}{10 \%} \quad \frac{\text { arcilla }}{10 \%}
$$

y añadir los análisis de los materiales de adición

\begin{tabular}{lllll} 
& $\mathbf{c}(\%)$ & s (\%) & a (\%) & f (\%) \\
\hline cal & 71,60 & 17,60 & 5,30 & 1,24 \\
arcilla & 53,50 & 30,00 & 9,35 & 3,10
\end{tabular}

a los análisis completos de la explotación no regulada. Las cifras están reunidas en la tabla 2 .

$$
\text { T A B L A } 2
$$

\begin{tabular}{|c|c|c|c|c|c|c|c|c|c|c|c|c|}
\hline & \multicolumn{2}{|c|}{$\underset{\substack{\text { Muestra } \\
\text { tomada azar }}}{\text { aza }}$} & \multicolumn{2}{|c|}{$\begin{array}{c}2 \\
\text { Muestra } \\
\text { tomada al } \\
\text { azar }\end{array}$} & \multicolumn{2}{|c|}{$\begin{array}{c}3 \\
\text { Muestra } \\
\text { tomada al } \\
\text { azar }\end{array}$} & \multicolumn{2}{|c|}{$\underset{\substack{\text { Muestra } \\
\text { tomada }}}{4}$ al } & \multicolumn{2}{|c|}{$\begin{array}{c}5 \\
\text { Muestra } \\
\text { tomada al } \\
\text { azar }\end{array}$} & \multicolumn{2}{|c|}{$\begin{array}{c}6 \\
\text { Muestra } \\
\text { tomada al } \\
\text { azar }\end{array}$} \\
\hline & $\overline{\mathbf{x}}$ & $\mathbf{s}$ & $\vec{x}$ & $\mathbf{s}$ & $\overline{\mathbf{x}}$ & $\mathbf{s}$ & $\overline{\mathbf{x}}$ & $\mathbf{s}$ & $\overline{\mathbf{x}}$ & $\mathbf{s}$ & $\bar{x}$ & $\mathbf{s}$ \\
\hline c & 67,52 & 2,16 & 67,51 & 2,18 & 68,01 & 1,66 & 67,61 & 1,79 & 67,51 & 1,93 & 67,21 & 2,27 \\
\hline $\mathrm{s} \quad\left(^{*}\right)$ & 19,87 & 1,545 & 19,86 & 1,510 & 20,26 & 0,955 & 20,57 & 1,010 & 20,67 & 1,06 & 19,78 & 1,29 \\
\hline$a$ & 6,57 & 0,394 & 6,57 & 0,410 & 6,68 & 0,255 & 6,72 & 0,280 & 6,61 & 0,319 & 6,62 & 0,372 \\
\hline $\mathrm{f}$ & 2,85 & 0,169 & 2,84 & 0,108 & 2,71 & 0,119 & 2,74 & 0,162 & 2,69 & 0,164 & 2,72 & 0,249 \\
\hline
\end{tabular}

Análisis y sus dispersiones en la explotación no regulada

(*) concentración de $\mathrm{SiO}_{2}$. 
De acuerdo con Linder (5) se define una suma de los cuadrados de los errores entre las muestras tomadas al azar:

$$
\mathrm{D}=\sum_{\mathrm{j}=1}^{\mathrm{M}} \mathrm{N}_{\mathrm{j}}\left(\overline{\mathrm{x}}_{\mathrm{j}}-\overline{\mathrm{x}}\right)
$$

lo cual indica lo mucho que divergen los valores medios aislados $\overline{\mathbf{x}}_{\mathrm{j}}$ del valor medio $\overline{\mathrm{x}}$ de la muestra tomada al azar unificada. En la explotación no regulada resulta, por ejemplo, para la concentración de $\mathrm{SiO}_{2}$ de la tabla 2:

$$
\begin{aligned}
\mathrm{Du}= & 100\left[(19,87-20,17)^{2}+(19,86-20,17)^{2}+(20,26-20,17)^{2}+(20,57-20,17)^{2}+\right. \\
& \left.+(20,67-20,17)^{2}+(19,78-20,17)^{2}\right]=99,41 .
\end{aligned}
$$

Para la explotación regulada se toma de la tabla 1 :

$$
\begin{aligned}
\text { Dg }= & 100\left[(20,83-20,79)^{2}+(20,86-20,79)^{2}+(20,70-20,79)^{2}+(20,74-20,79)^{2}+\right. \\
& \left.+(20,78-20,79)^{2}+(20,86-20,79)^{2}\right]=2,21 .
\end{aligned}
$$

Si la suma de los cuadrados de los errores entre las pruebas al azar según la ecuación [11] es notablemente mayor que la suma de los cuadrados de los errores dentro de las pruebas al azar:

$$
S_{x x}=\sum_{j=1}^{M} S_{x x}^{(j)}
$$

(en la cuai $\mathrm{S}_{\mathrm{xx}}^{(\mathrm{j})}$ es la suma de los cuadrados de los errores de la prueba al azar $\mathrm{j}$ ), lo que representa una suma de los cuadrados de los errores de las diversas pruebas al azar, los grupos se mantienen debidamente separados.

En los dos casos que interesan resulta:

$1 .^{\circ}$ para la explotación no regulada:

$$
\mathrm{S}_{\mathrm{xx}}^{(\mathrm{u})}=929,3031
$$

2. ${ }^{\circ}$ para la explotación regulada:

$$
\mathrm{S}_{\mathrm{xx}}^{(\mathrm{g})}=445,7772
$$

El límite que indica si las muestras tomadas al azar hay que separarlas o que reunirlas, se determina por el cociente:

$$
\mathrm{F}=\frac{\mathrm{D} /(\mathrm{M}-1)}{\mathrm{S}_{\mathrm{xx}} /(\mathrm{N}-\mathrm{M})}
$$

Este cociente $\mathrm{F}$ está distribuido con los grados de libertad:

$$
\mathrm{f}_{1}=\mathrm{M}-1 \mathrm{y} \mathrm{f}_{2}=\mathrm{N}-\mathrm{M} \text {. }
$$

Las distribuciones $\mathrm{F}$ están reunidas para diversos grados de confianza estadísticos y grados de libertad, en forma de tablas en libros competentes de la estadística matemática (3). Si el valor $\mathrm{F}$ calculado es mayor que el fractil $\mathrm{F}$ respectivo de la tabla, se mantienen 
las muestras tomadas al azar debidamente separadas. En caso contrario, pueden reunirse en una muestra al azar colectiva.

Para la explotación no regulada se calcula:

y para la explotación regulada:

$$
\mathrm{F}_{\mathrm{u}}=10,6
$$

$$
\mathrm{F}_{\mathrm{g}}=0,49 \text {. }
$$

El fractil tiene el valor $\mathrm{F}=2,2$ con $95 \%$ de grado de confianza estadística.

Como se observa claramente, en la explotación no regulada no deben de reunirse las muestras tomadas al azar en una muestra colectiva, pero en cambio sí en la explotación regulada. Como resultado de estas consideraciones, queda por determinar si las fluctuaciones, de larga duración - que significan lo mismo que valores medios diferentes de sucesivas muestras tomadas al azar- pueden eliminarse bien de la regulación.

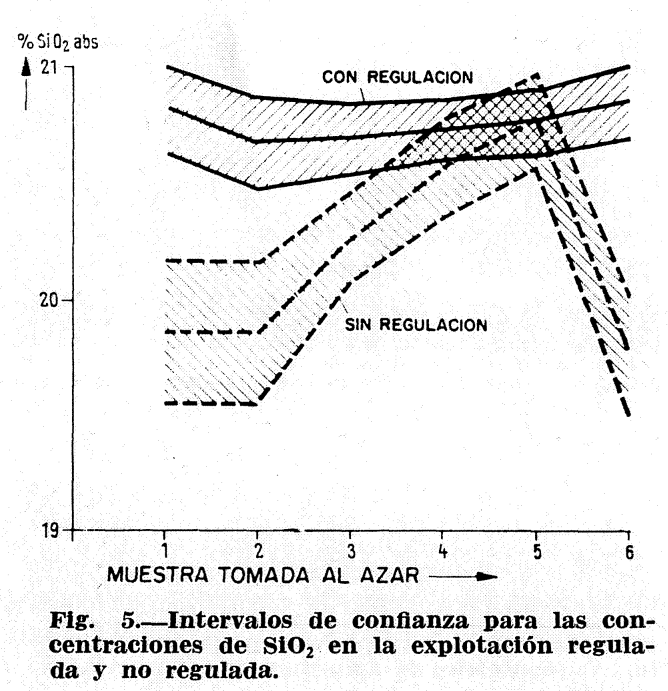

También los intervalos de confianza muestran claramente (fig. 5) para las 6 muestras tomadas al azar, las diferencias de la explotación no regulada:

1. los intervalos de confianza son mayores;

2. ${ }^{\circ}$ los valores medios divergen mucho más unos de otros;

$3 .^{\circ} \quad$ los intervalos de confianza se cubren sólo para dos muestras tomadas al azar parcialmente.

Parecidas consideraciones pueden hacerse para las otras tres concentraciones. Pero no aportan ningún conocimiento nuevo y por tal motivo no se indican aquí.

Las mejoras en la explotación regulada parecen ser insignificantes al principio. Pero hay

que tener en cuenta que la concentración de $\mathrm{SiO}_{2}$ entra con el coeficiente 2,8 en el cálcula del standard de la cal y que la concentración de $\mathrm{CaO}$ acusa frecuentemente la tendencia contraria. Para el propio standard de la cal, que representa la verdadera característica de una mezcla de crudo, son, por consiguiente, mucho más acusadas las diferencias reseñadas en la figura 5.

\subsection{Curso temporal y análisis de Fourier}

La figura 6 representa el curso temporal de la concentración de $\mathrm{SiO}_{2}$ de 128 muestras de media hora, tomadas en el punto de toma 1.

Junto al curso en la explotación regulada se representa el curso en la explotación no regulada. En la figura se ve que las fluctuaciones son notables y que se pueden distinguir muy bien las concentraciones de dos valores sucesivos. Las amplitudes con regulación son menores, no representándose las "irrupciones" extremas en la explotación no regulada. 
Como demostración, se ha incluido todavía el standard de cal para la explotación regulada. Acusa un curso contrapuesto a la concentración de $\mathrm{SiO}_{2}$ y graba las fluctuaciones de la proporción de $\mathrm{SiO}_{2}$ por la gran sensibilidad de este módulo a las variaciones del citado óxido.
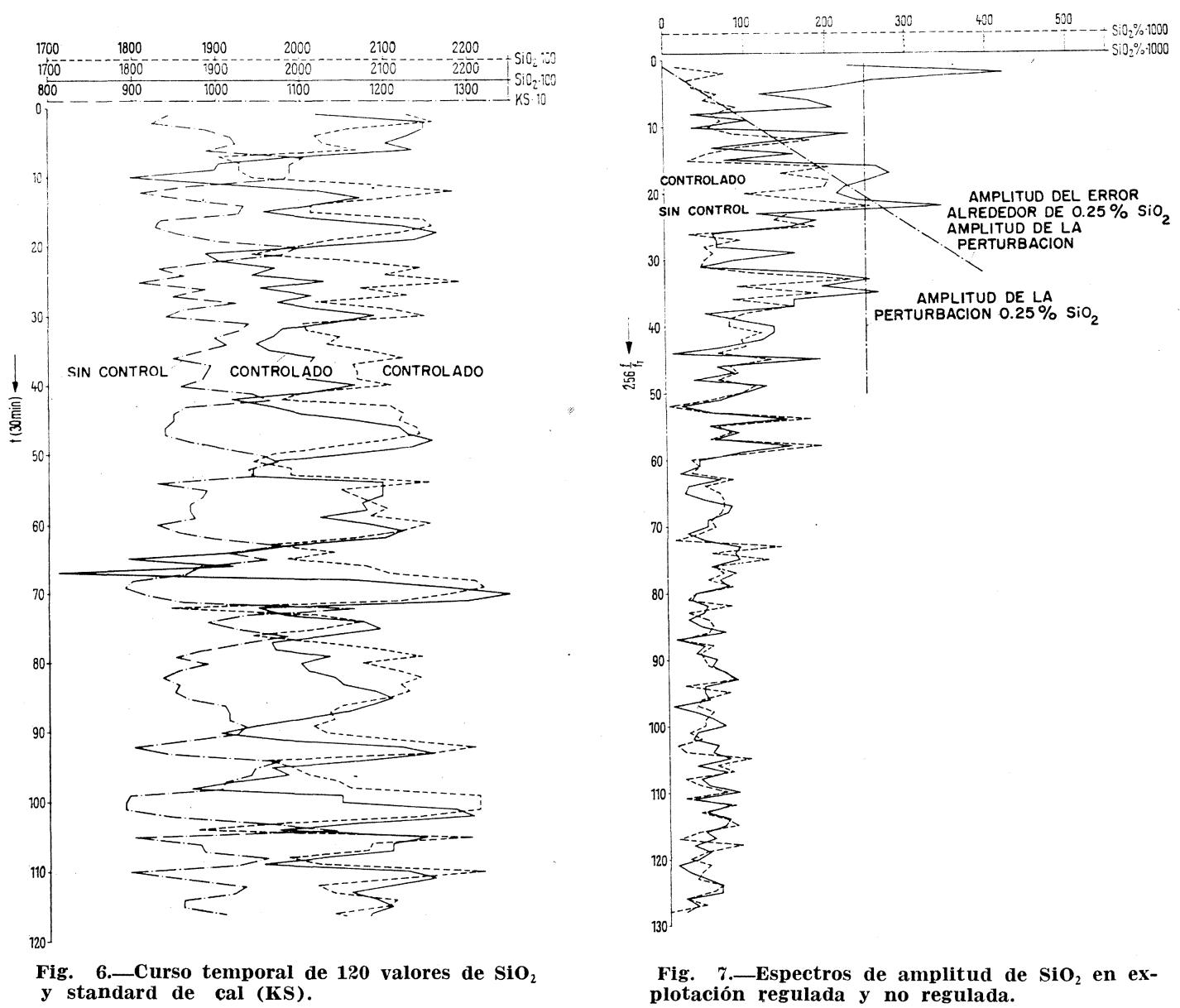

Pero el curso temporal no permite obtener conclusiones definitivas sobre la calidad de la regulación.

Por esta causa, el curso temporal de 256 concentraciones de $\mathrm{SiO}_{2}$ para explotación regulada y no regulada se sometió al análisis de Fourier, que como se sabe descompone el curso en un número de oscilaciones senoidales.

Si en general se designa con $\mathrm{x}_{\mathrm{j}}$ un punto del curso temporal según la figura 6, para las amplitudes de las oscilaciones senoidales y cosenoidales $b_{\mathrm{k}}$ o bien $a_{\mathrm{k}}$ sirven las relaciones:

$$
\begin{aligned}
& a_{\mathrm{k}}=\frac{1}{\mathrm{n}} \sum_{\mathrm{j}=0}^{2 \mathrm{n}-1} \mathrm{x}_{\mathrm{j}} \cos (\pi \mathrm{jk} / \mathrm{n}), \text { para } \mathrm{k}=0,1, \ldots \ldots, \mathrm{n} \\
& \mathrm{b}_{\mathrm{k}}=\frac{1}{\mathrm{n}} \sum_{\mathrm{j}=0}^{2 \mathrm{n}-1} \mathrm{x}_{\mathrm{j}} \operatorname{sen}(\pi \mathrm{jk} / \mathrm{n}), \text { para } \mathrm{k}=0,1, \ldots \ldots, \mathrm{n}
\end{aligned}
$$

donde $2 \mathrm{n}$ es el número de valores de la función. 
Las oscilaciones senoidales y cosenoidales de igual frecuencia pueden reunirse en una oscilación compleja, cuya amplitud $\mathrm{c}_{\mathrm{k}}$ se puede calcular con la ecuación [16]:

$$
c_{\mathrm{k}}=\sqrt{{a_{\mathrm{k}}{ }^{2}+\mathrm{b}_{\mathrm{k}}{ }^{2}}}
$$

Las amplitudes $c_{k}$ de un análisis de Fourier semejante de las concentraciones de $\mathrm{SiO}_{2}$ d ll crudo en la explotación no regulada y en la regulada para 256 valores funcionales a distancias temporalmente iguales, se representan en la figura 7. En el eje de la abscisa se ha indicado en $\%$ la concentración de $\mathrm{SiO}_{2}$ como función de la frecuencia de toma de muestras $f_{T}$ que se representa en el eje de ordenadas.

En la gama inferior de frecuencia $\left(0,0039 \mathrm{f}_{\mathrm{T}} \ldots \ldots \ldots 0,039 \mathrm{f}_{\mathrm{T}}\right)$, la amortiguación de las amplitudes es buena debido a la regulación. En frecuencias más elevadas ya no cumple la regulación. Ya no se amortiguan las amplitudes perturbadoras, y tanto las magnitudes reguladas como las no reguladas acusan prácticamente las mismas amplitudes.

Hay que advertir de todas formas que 256 puntos son relativamente pocos para el espectro de una magnitud casi sin regulación, como la que representa el curso de la concentración de $\mathrm{SiO}_{2}$. Por lo tanto, las amplitudes del espectro acusan una cierta inseguridad. No obstante, los resultados debieran de mostrar lo esencial de la explotación regulada. Suponen un complemento de la dispersión según el apartado 3.23 y confirman estos resultados.

\subsection{Errores dinámicos de la regulación}

En el apartado anterior se mostró que las amplitudes de la regulación se amortiguan cada vez peor al aumentar la frecuencia. Una consideración aproximada permite estimar los errores dinámicos de la regulación. Con este fin en la figura 8 se representa el circuito de regulación de una concentración. El comportamiento dinámico del molino se ha aproximado mediante un sencillo elemento de tiempo de funcionamiento, cuyo tiempo de funcionamiento es algo menos que el retículo de toma de muestras T. El computador como regulador actúa aproximadamente como un pulsador ideal con integrador ideal como acumulador. Un análisis del circuito de regulación se consigue con ayuda de la transformación de z. Pero aquí bastan representaciones gráficas para reconocer lo esencial.

La figura 9 representa el curso de la divergencia de regulación con distintas señales perturbadoras. En una perturbación a modo de saltos, aparece el error inmediatamente antes de la toma de muestras en el momento $\mathrm{T}$, queda parado durante un ciclo debido al tiempo muerto del molino y se compensa seguidamente por el regulador.

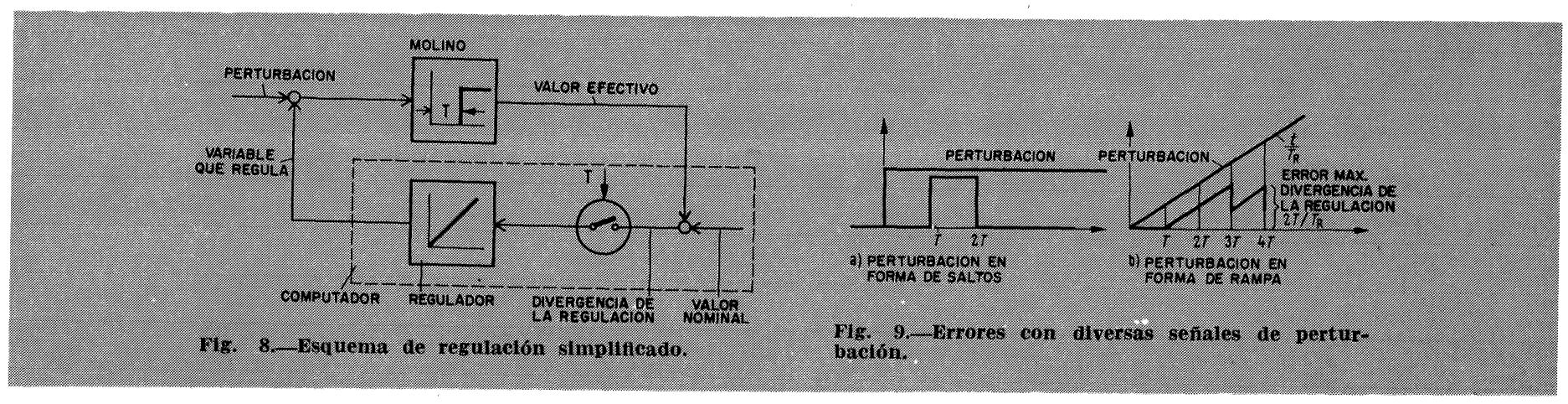


Con curso en forma de rampa de las magnitudes perturbadoras se presenta después de un ciclo de toma de muestras un error que puede no tenerse en cuenta; el computador toma entonces sus contramedidas. Después de $2 \mathrm{~T}$ sigue un orden de regulación de acuerdo con la divergencia de regulación $\mathrm{T} / \mathrm{T}_{\mathrm{R}}$ medida. Como consecuencia del tiempo inefectivo del molino aumenta sin embargo la amplitud todavía hasta el momento $3 \mathrm{~T}$ a $2 \mathrm{~T} / \mathrm{T}_{\mathrm{R}}$, entonces salta el error hasta el valor $\mathrm{T} / \mathrm{T}_{\mathrm{R}}$ debido al desplazamiento al momento $2 \mathrm{~T}$. Hasta el momento $4 \mathrm{~T}$ vuelve a aumentar a $2 \mathrm{~T} / \mathrm{T}_{\mathrm{R}}$.

Se ve que la regulación hace que el error (divergencia de la regulación) sea cero en perturbaciones a modo de saltos, pero que no lo puede compensar completamente. El error depende de la pendiente de la rampa $1 / \mathrm{T}_{\mathrm{R}}$ y tiene como máximo el valor:

$$
\mathrm{E}_{\max }=2 \mathrm{~T} / \mathrm{T}_{\mathrm{R}}
$$

Oscilaciones senoidales tienen, como es sabido, su máxima pendiente en la pasada cero y pueden ser aproximadas allí por medio de una recta. Estas propiedades permiten aplicar la ecuación [17]. Si se supone que una concentración $\mathrm{SiO}_{2}$ max varía en forma senoidal con la frecuencia $f$ :

$$
\mathrm{SiO}_{2}=\mathrm{SiO}_{2 \text { max }} \operatorname{sen}(2 \pi \mathrm{ft})
$$

tiene la velocidad máxima de variación:

$$
\mathrm{SiO}_{2 \mathrm{~m}}^{*}=\mathrm{SiO}_{2 \max } 2 \pi \mathrm{f}
$$

Si es $\mathrm{f} \ll \mathrm{f}_{\mathrm{T}}$-la frecuencia de la toma de muestras-, f se mueve por ejemplo entre $0 \leqslant f \leqslant \frac{1}{8} f_{T}$, resulta entonces de las ecuaciones [17] y [19]:

$$
\begin{aligned}
\mathrm{E}_{\max } & =\frac{4 \pi \mathrm{SiO}_{2 \max }}{\alpha} \\
\alpha & =\mathrm{f} / \mathrm{f}_{\mathrm{T}}
\end{aligned}
$$

Para una amplitud constante de la perturbación, por ejemplo $0,25 \% \mathrm{SiO}_{2}$, también se ha incluido este error en la figura 7. Los valores teóricos suministran una buena estimación de las amplitudes de error.

\section{EL ANALISIS DESPUES DEL SILO DE ALMACENAMIENTO}

Como se ve por la figura 1, el crudo llega del molino al silo de almacenamiento, del que se extrae para el horno de acuerdo con las necesidades. En el silo de almacenamiento prácticamente ya no se homogeneiza, sólo se le mueve con aire comprimido para hacer un vaciado exento de interrupciones.

Las muestras del punto de toma 3 (fig. 1), extraídas de hora en hora, únicamente determinan con qué amortiguaciones hay que contar a través del silo de almacenamiento.

En las figuras 2 y 3 se han dibujado las gamas de dispersión para $\mathrm{SiO}_{2}$ o $\mathrm{CaO}$, después del silo de almacenamiento, de las cuales puede calcularse un coeficiente medio de amor- 
tiguación de 2,5 para el silo. Indica como se reduce por el silo la dispersión de una concentración a la salida del molino.

Queda por señalar como, sobre todo las rápidas oscilaciones, que no pueden captarse por la regulación de la mezcla, son amortiguadas por el silo de almacenamiento; en cambio, las oscilaciones de larga duración del material, no pueden ser absorbidas por el silo.

La tabla 3 indica, en forma similar a la tabla 1 , tres muestras tomadas al azar, cada una con 100 análisis aislados del punto de toma 3. Las diferencias en los valores medios del standard de cal entre la muestra tomada al azar n. ${ }^{\circ} 1$ y las otras dos muestras, tenían su origen en otro valor teórico $\mathrm{KS}_{\mathrm{nom}}$ para la molienda en este silo.

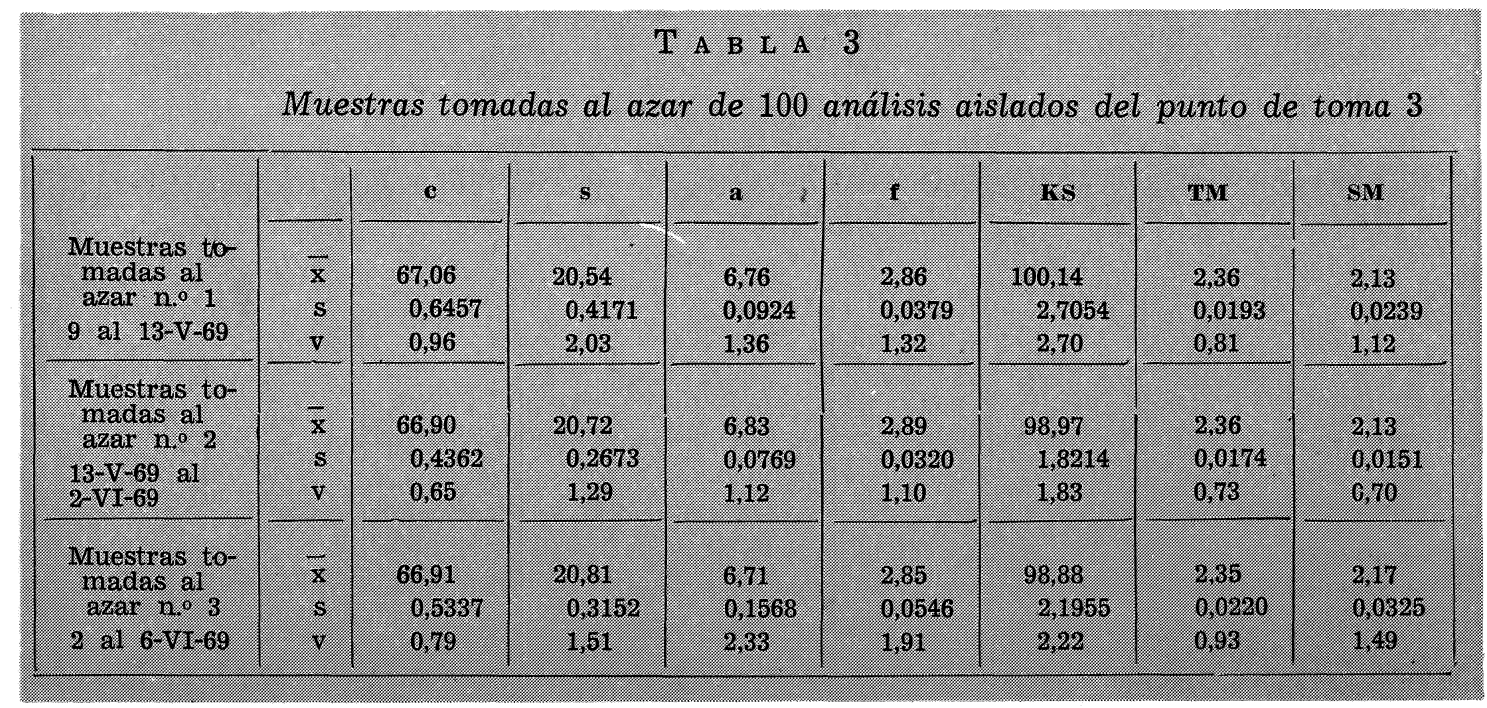

\section{PRECISION DEL ESPECTROMETRO MULTICANAL DE RAYOS $\mathbf{X}$}

\subsection{Examen de las oscilaciones de larga duración mediante corrección de curvas de calibrado}

El expectrómetro de rayos $\mathrm{X}$ suministra a las concentraciones los correspondientes grupos de impulsos (IR). En el caso más sencillo se hallan ligados recíprocamente grupos de impulsos y concentración $\mathrm{C}$ a través de una relación lineal de la forma:

$$
\mathrm{C}=\alpha+\beta \cdot \mathrm{IR}
$$

Una tableta standard de composición química conocida suministra de acuerdo con el envejecimiento, por ejemplo oscilación de larga duración del espectrómetro de rayos $\mathrm{X}$ de larga duración, diversos grupos (contingentes) de impulsos para una concentración constante. Estas oscilaciones influyen en la medida de paso $\beta$ de la ecuación de la curva de contraste [22], siendo esta influencia reducida. La sección del eje $\alpha$ varía en primer lugar con estas oscilaciones y en la práctica se corrige sólo de acuerdo con la relación siguiente $(1)$ :

$$
\alpha_{1}=\alpha_{0}+\left(\mathrm{C}_{2}-\mathrm{C}_{1}\right)
$$

Si primeramente se analiza la tableta standard y hasta poco después no se efectúa la co- 
rrección de la curva de contraste, una serie de análisis de este tipo da una medida para las oscilaciones de larga duración del MRS.

La tabla 4 muestra 3 muestras tomadas al azar de 100 análisis aislados cada una, como las que se obtuvieron con arreglo al método descrito.

La muestra 1 indica el caso normal. En cambio, la muestra 2 acusa una dispersión notablemente mayor en el standard de cal. Fue originada por una avería en el abastecimiento de alta tensión del MRS, como pudo comprobarse después. La muestra 3 acusa también aproximadamente $50 \%$ más de dispersión que la muestra 1 . En este caso se pudo comprobar que una corrección de la curva de contraste no pudo realizarse con la tableta standard, sino con una muestra del punto de toma 1 . De este modo sencillo se pudieron observar con el computador irregularidades en el MRS o bien en su manejo.

T A B L A 4

Muestras tomadas al azar de 100 análisis aislados para la corrección de la curva de calibrado

\begin{tabular}{|c|c|c|c|c|c|c|c|c|}
\hline & & c & s & $\mathbf{a}$ & f & $\mathbf{K S}$ & $\mathbf{T M}$ & SM \\
\hline $\begin{array}{l}\text { Muestras to- } \\
\text { madas al } \\
\text { azar n. } 1 \\
9 \text { al } 13-V-69\end{array}$ & $\begin{array}{l}\bar{x} \\
s \\
v\end{array}$ & $\begin{array}{l}66,26 \\
0,1964 \\
0,29\end{array}$ & $\begin{array}{c}20,94 \\
0,1069 \\
0,51\end{array}$ & $\begin{array}{l}7,05 \\
0,0720 \\
1,02\end{array}$ & $\begin{array}{l}2,94 \\
0,0214 \\
0,72\end{array}$ & $\begin{array}{l}96,74 \\
0,6913 \\
0,71\end{array}$ & $\begin{array}{l}2,39 \\
0,0150 \\
0,62\end{array}$ & $\begin{array}{l}2,09 \\
0,0163 \\
0,77\end{array}$ \\
\hline $\begin{array}{l}\text { Muestras to- } \\
\text { madas al } \\
\text { azar n. } 2 \\
13-V-69 \text { al } \\
3-\mathrm{VI}-69\end{array}$ & $\begin{array}{l}\bar{x} \\
\text { s } \\
\text { v }\end{array}$ & $\begin{array}{c}66,26 \\
0,6167 \\
0,933\end{array}$ & $\begin{array}{c}20,95 \\
0,1567 \\
0,75\end{array}$ & $\begin{array}{l}7,05 \\
0,0911 \\
1,29\end{array}$ & $\begin{array}{l}2,94 \\
0,0274 \\
0,93\end{array}$ & $\begin{array}{c}96,72 \\
1,1177 \\
1,155\end{array}$ & $\begin{array}{l}2,39 \\
0,0173 \\
0,725\end{array}$ & $\begin{array}{l}2,09 \\
0,0297 \\
1,42\end{array}$ \\
\hline $\begin{array}{l}\text { Muestras to- } \\
\text { madas al } \\
\text { azar n. } 3 \\
3 \text { al } 8 \text { VI-69 }\end{array}$ & $\begin{array}{l}\bar{x} \\
s \\
v\end{array}$ & $\begin{array}{l}66,25 \\
0,2298 \\
0,34\end{array}$ & $\begin{array}{l}20,95 \\
0,1387 \\
0,52\end{array}$ & $\begin{array}{l}7,05 \\
0,0744 \\
1,05\end{array}$ & $\begin{array}{l}2,94 \\
0,0264 \\
0,89\end{array}$ & $\begin{array}{c}96,70 \\
0,9043 \\
0,91\end{array}$ & $\begin{array}{l}2,39 \\
0,0151 \\
0,63\end{array}$ & $\begin{array}{l}2,09 \\
0,0137 \\
0,65\end{array}$ \\
\hline
\end{tabular}

La comparación siguiente de los 3 valores medios del standard de cal con 100 análisis consecutivos cada uno, con el standard de cal del análisis químico:

\begin{tabular}{cc}
$\begin{array}{c}\text { Análisis } \\
\text { químico } \\
\text { KS }\end{array}$ & $\begin{array}{c}\text { Valores medios } \\
\text { de los análisis } \\
\text { de corrección } \\
\text { KS }\end{array}$ \\
\cline { 2 - 2 } 96,74 & 96,74 \\
& 96,72 \\
& 96,70
\end{tabular}

hace que se reconozca que los valores medios son menores o como máximo iguales al valor del análisis químico. La corrección se efectúa por lo tanto únicamente en una dirección, caso contrario tendría que haber también valores sobre el valor del análisis químico. Este fenómeno que se ha descrito es típico para una "corriente", que puede señalar envejecimiento de determinados elementos constructivos

\subsection{Las oscilaciones de corta duración y precisión de medidas que pueden conseguirse con el MRS}

Cada medida que se efectúa después de una corrección de la curva de contraste posee la máxima precisión que se puede conseguir, ya que dentro de este pequeño lapso de tiem- 
po no llaman la atención oscilaciones de larga duración. Si la misma muestra se vuêlve a medir siempre después de una corrección de la curva de contraste, se llegan a obtener afirmaciones sobre las oscilaciones de corta duración del MRS. La estadística de dos muestras tomadas al azar de 100 mediciones de este tipo cada una, se ve en la tabla 5. La precisión máxima que puede conseguirse con $95 \%$ de seguridad estadística se encuentra, aproximadamente, en $\pm 0,5$ puntos en el standard de la cal. Hay que tener en cuenta que estas precisiones valen para análisis de material crudo sin efecto matriz. Sobre la pérdida de precisión con efecto matriz, nos remitimos a los trabajos de F. Henkel (7). En comparación con las dispersiones de los análisis de los puntos de toma 1 y 3 , esta precisión es suficiente.

\begin{tabular}{|c|c|c|c|c|c|c|c|c|}
\hline \multicolumn{9}{|c|}{ Muestras tomadas al azar, cada una de 100 determinaciones, para determinación } \\
\hline \multirow[b]{2}{*}{$\begin{array}{l}\text { Muestras to- } \\
\text { madas al } \\
\text { azar n. } 1\end{array}$} & & c & 8 & a. & f & KS & $\mathrm{TMI}$ & SMI \\
\hline & s & $\begin{array}{l}66,35 \\
0.0792 \\
0.11\end{array}$ & 20.97 & $\begin{array}{l}7.066 \\
0,0281 \\
0,39\end{array}$ & $\begin{array}{l}2,96 \\
0,0099 \\
0,39\end{array}$ & $\begin{array}{l}96,72 \\
0,2476\end{array}$ & $\begin{array}{l}2,38 \\
0,0127 \\
0,58\end{array}$ & $\begin{array}{l}2.09 \\
0.0094 \\
0.44\end{array}$ \\
\hline 9 al $18-V-69$ & V. & & & & & & & \\
\hline 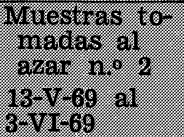 & s. & $\begin{array}{l}66,33 \\
0,0748 \\
0,11\end{array}$ & $\begin{array}{l}20,98 \\
0,0556 \\
0,26\end{array}$ & $\begin{array}{l}7.07 \\
0.0325 \\
0.46\end{array}$ & $\begin{array}{l}2,96 \\
0,0111 \\
0,37\end{array}$ & $\begin{array}{l}96,63 \\
0,2503 \\
0,25\end{array}$ & $\begin{array}{l}2,38 \\
0,0127 \\
0,58\end{array}$ & $\begin{array}{l}2,09 \\
0,0099 \\
0,47\end{array}$ \\
\hline
\end{tabular}

\section{OBSERVACION FINAL}

No obstante el pequeño gasto en la homogeneización de la piedra y del crudo, el computador permite captar las oscilaciones extremas en la piedra cruda a través de la regulación de la mezcla y compensar en forma casi ideal las oscilaciones de larga duración.

\section{R E S U M E N}

A base de amplio material numérico estadístico, se examina la precisión de la regulación de una instalación de molienda de crudo. Valores medios y dispersiones no describen de manera fehaciente la precisión de la regulación. El análisis de la dispersión y el análisis de Fourier suministran resultados que concuerdan bien con los obtenidos teóricamente.

Mediante análisis repetido de una muestra patrón, puede indicarse la dispersión a corto y largo plazo del espectrómetro multicanal de rayos $\mathrm{X}$. La precisión que se consigue es suficiente para la explotación.

\section{B I B L I O G R A F I A}

1) Gerstberger, R.: Métodos estadisticos en la valoración de muestras para el análisis del crudo y su aplicación práctica en la preparación de crudos mediante computador de procesos. Zement-Kaldk-Gips 23 (1970), 12.

(2) Gerstberger, R.: Computadores de procesos en fábricas de cemento. Siemens-Zeitschrift 42 (1968), n. ${ }^{\circ} 9,673-679$.

(3) Wackerle, H.: Mando de los procesos EVD en fábricas de cemento. Maschinenmarkt, n. ${ }^{\circ}$ 90, noviembre 1966, 1966-1969.

(4) ZURMüHL, R.: Matemáticas prácticas. Springer 1965.

(5) LINDER, A.:Métodos estadisticos. Birkhäuser, Basilea 1965.

(6) Henkel, F.: Aplicación del análisis de fluorescencia de rayos $X$ en el laboratorio de láminas. ZementKalk-Gips, 18 (1965), 253-258.

(7) Henkel, F.: Análisis por fluorescencia de rayos $X$ de los crudos de cemento. Zement-Kalk-Gips, 22 (1969), 5-9. 